\title{
Characterization of the energy-dependent uncertainty and correlation in silicon neutron displacement damage metrics
}

\author{
Patrick Griffin ${ }^{1, a}$, Dimitri Rochman ${ }^{2}$, and Arjan Koning ${ }^{3}$ \\ 1 Sandia National Laboratories, Albuquerque, USA \\ 2 Paul Scherrer Institut, Villigen, Switzerland \\ 3 International Atomic Energy Agency, Vienna, Austria
}

\begin{abstract}
A rigorous treatment of the uncertainty in the underlying nuclear data on silicon displacement damage metrics is presented. The uncertainty in the cross sections and recoil atom spectra are propagated into the energy-dependent uncertainty contribution in the silicon displacement kerma and damage energy using a Total Monte Carlo treatment. An energy-dependent covariance matrix is used to characterize the resulting uncertainty. A strong correlation between different reaction channels is observed in the high energy neutron contributions to the displacement damage metrics which supports the necessity of using a Monte Carlo based method to address the nonlinear nature of the uncertainty propagation.
\end{abstract}

\section{Introduction}

The focus of this paper is on a rigorous quantification of the uncertainty in silicon displacement damage metrics due to the uncertainty in the underlying nuclear data. Silicon displacement damage metrics, such as the displacement kerma or the damage energy, are used in the assessment of the damage to the minority carrier lifetime in silicon bipolar junction transistors (BJTs). This is an important damage metric to the radiation effects community because of: 1) the wide-spread use of silicon in radiation detectors and as a radiation environment diagnostic; 2) the existence of a radiation effects community endorsed standard that addresses the proper calculated metric to use for correlating radiation damage in different neutron fields, i.e., the ASTM E722 Standard Practice for Characterizing Neutron Fluence Spectra in Terms of an Equivalent Monoenergetic Neutron Fluence for Radiation-Hardness Testing of Electronics [1]. This response function is also recommended as a direct dosimetry metric in ASTM E1855-15 Standard Test Method for Use of 2N2222A Silicon Bipolar Transistors as Neutron Spectrum Sensors and Displacement Damage Monitors [2]. This ASTM standard supports the use of this response function, in conjunction with a silicon bipolar transistor, for both the direct characterization of the damage metric for silicon electronics in neutron fields and as a sensor appropriate for use in spectrum adjustments/unfolds in order to cover an energy region in research reactors that is not easily covered by non-fission based dosimetry sensors. The ability to perform this rigorous analysis is supported by the availability and high quality of the important supporting nuclear data in the naturally occurring silicon isotopes.

This paper uses a Total Monte Carlo approach [3] to capture the nonlinear propagation of the uncertainty in the nuclear data into the damage metrics. It characterizes the

a e-mail: uthor@e-mail.org uncertainty of the silicon displacement kerma in the form of an energy-dependent covariance matrix that can be used to assess various material damage studies in support of the use of silicon transistors as a neutron dosimeter in accordance with ASTM standards E1855 and E722.

The following sections address: a) the definition of the calculated radiation damage metrics that can be correlated with the observed damage mechanism; b) an examination of the aspects of uncertainty in the nuclear data that affect the calculated metric; and c) the approach used to obtain, for these damage metrics, a rigorous quantification of the neutron energy-dependent uncertainty in the form of a covariance matrix.

\section{Neutron displacement damage metrics}

The fundamental calculated displacement damage metric is the microscopic displacement kerma factor, $\kappa^{d p a}$, see ASTM E722-14 [1], which can be written as:

$$
\begin{aligned}
\kappa^{d p a}(E)= & \sum_{i, j_{i}} \sigma_{i, j_{i}}(E) \int_{0}^{\infty} d T_{R, j_{i}} \int_{-1}^{1} d \mu \cdot f_{i, j_{i}} \\
& \times\left(E, \mu, T_{R, j_{i}}\right) \cdot{ }^{i o n} T_{\text {dam }}\left(T_{R, j_{i}}\right) .
\end{aligned}
$$

where:

- the summation is over all open neutron-induced reaction channels, $\mathrm{i}$, and emitted particles $\mathrm{j}_{i}$

- $\sigma_{i, j i}(E)$ is the cross section for the $\mathrm{j}_{i}$ particle in the $\mathrm{i}^{\text {th }}$ reaction channel

- $f_{i, j i}\left(E, \mu, T_{R, j i}\right)$ is the energy distribution for resulting charged particles which are emitted with:

a recoil ion energy $\mathrm{T}_{R, j i}$ at an angle characterized by $\mu$ and resulting from the $\mathrm{j}_{i}$ particle in the $\mathrm{i}^{\text {th }}$ channel,

o induced by the incident neutron energy with energy E. 
- ${ }^{i o n} T_{d a m}\left(T_{R, j i}\right)$ is the displacement energy partition function for the emitted recoil ion $\mathrm{j}_{i}$ in the $\mathrm{i}^{\text {th }}$ open channel with recoil energy $T_{R, j i}$.

The displacement kerma, $\mathrm{K}$, is equal to the microscopic kerma factor multiplied by the number of atoms per unit mass in the target material and the incident particle fluence. The ionizing kerma can be obtained by subtracting the displacement kerma from the total kerma.

A related, but very important, damage metric used by the radiation effects community is the neutron damage energy, ${ }^{n} \mathrm{~T}_{\text {dam }}$, however, the definition of damage energy is not entirely consistent throughout the literature [4]. The variation in the use of the term involves the: 1) selection of the lower energy bound for the integration over recoil ion energies that are to represent interactions where a subsequent lattice atom displacement can occur; 2) selection of the displacement model, which involves identifying a transfer energy associated with the generation of a Frenkel pair; and 3) treatment of the effective deposited energy for ion recoil energies near the threshold displacement energy. The concept of a "damage energy" is intended to capture the energy imparted by secondary charged particles, integrated over a defined portion of the slowing down process, and going into the creation of Frenkel pairs.

The most commonly used form of the neutron damage energy has a lower recoil energy integration bound, a bound for the integral over the recoil ion energy, set to the threshold displacement energy, $E_{d}$, and uses a sharp threshold Kinchin-Pease displacement model [5] with the Robinson damage partition function [6]. $E_{d}$ is intended to represent an emission angle-integrated average recoil ion energy below which the recoiling atom can no longer result in a displacement of any additional lattice atoms. This selection is labeled as a "threshold-based damage energy".

The determination of the uncertainty in the calculated damage energy requires that we look at the energydependent uncertainty in the various quantities in Eq. (1). This energy-dependent uncertainty is best captured as a covariance function, which is equivalent to providing an energy-dependent standard deviation and a correlation matrix. It needs to be noted that the higher moments in a distribution are not captured in a covariance representation, so a more rigorous approach is always to use a full TMC approach all the way through the calculation of the desired integral damage metric.

The major components within the integrand of Eq. (1) are: a) the reaction cross section and resulting recoil ion spectra; b) the partition function that divides the recoil energy into an ionizing component and a non-ionizing component; and c) a threshold damage treatment. The focus of this paper is on a detailed examination of the uncertainty due to the first of these three uncertainty categories, i.e., the nuclear data. When this uncertainty contribution is expressed in the form of a covariance matrix, it can be combined with the uncertainty due to the other two categories. Since the uncertainty in these three categories are uncorrelated with each other, the uncertainty in the damage energy can then be expressed as the sum of the covariance matrices for the three categories.

\section{Uncertainty methodology}

An inspection of Eq. (1) shows that the nuclear data enters into the damage metric via the cross section term, $\sigma_{i, j i}(E)$, and the recoil spectra, $f_{i, j i}\left(E, \mu, T_{R, j i}\right)$. There is a summation over all reaction channels and an integral over the recoil spectrum as weighted by the damage partition function. An uncertainty methodology that addresses the nonlinear propagation of the uncertainty due to the nuclear data is required.

\subsection{Relative importance of reaction channels}

The approach adopted here is to use the NJOY-2012 [7] code, in conjunction with a nuclear data evaluation, to find the microscopic displacement kerma factor, $\kappa^{d p a}$. NJOY first calculates the reaction-specific total kerma induced by neutron interactions and then identifies the recoil energy distribution for all of the reaction products. The recoil atom energy distribution for each reaction channel is then partitioned into a displacement energy component using a damage partition function. The NJOY2012 code implements the Robinson fit [6] to the LSS theory [8] for the energy partition and applies a displacement threshold energy, $E_{d}$, as the lower integration limit over recoil ion energy. NJOY-2012, in normal operational mode, calculates this sharp threshold KinchinPease neutron damage energy [4]. The neutron damage energy is equivalent to the displacement kerma only when a displacement threshold energy of zero is used in the expression.

NJOY cannot just produce the total damage energy, but it can separately output the various components of the damage energy. Using the ENDF-6 format MT nomenclature for the various reaction channels, the NJOY code supports output of the total $[\mathrm{MT}=1]$, elastic $[\mathrm{MT}=2]$, inelastic $[\mathrm{MT}=51-91]$, and disappearance $[\mathrm{MT}=102-120]$ damage energy components. There is another component, one obtained by subtracting the elastic, inelastic and disappearance component from the total damage energy, that, for the ${ }^{28} \mathrm{Si}$ isotope, includes MT $=16,22,24,28$, and 29 channels, i.e., the $(n, 2 n)$, $(\mathrm{n}, \mathrm{n} \alpha),(\mathrm{n}, 2 \mathrm{n} \alpha),(\mathrm{n}, \mathrm{np})$ and $(\mathrm{n}, \mathrm{n} 2 \alpha)$ channels. Figure 1 shows the energy-dependent fractional contribution from each of these damage components. Figure 2 shows an enlarged view of the high energy portion of these contributions to the damage energy. The sharp dropoff in the Fig. 1 elastic channel fractional contribution to the damage energy, and the rise in the importance of the $(\mathrm{n}, \gamma)$ disappearance channel contribution, near $220 \mathrm{eV}$ corresponds to the neutron energy, $E$, where the kinematics of the elastic scattering result in a maximum silicon atom recoil energy, $T_{R, \text { elastic, }}$, that has a damage energy, ${ }^{i o n} T_{\text {dam }}\left(T_{R, \text { elastic }}\right)$, less than the silicon displacement threshold energy of $20.5 \mathrm{eV}$.

\subsection{Influence of recoil spectra}

The damage energy depends not just on the cross section, but also on the resulting recoil ion spectra. These recoil spectra are quite complex, varying with the incident neutron energy and the reaction channel. Figure 3 shows some representative recoil energy spectra for a $15-\mathrm{MeV}$ neutron on ${ }^{28} \mathrm{Si}$. Figures 4 and 5 show representative comparisons of the agreement of the recoil spectrum between different evaluated nuclear data files for the elastic and $(\mathrm{n}, \mathrm{n} \alpha)$ reaction channels with incident neutron energies of 10 and $20 \mathrm{MeV}$, respectively. From the broad comparison of different nuclear data evaluations, the agreement in recoil spectra is seen to be very good 


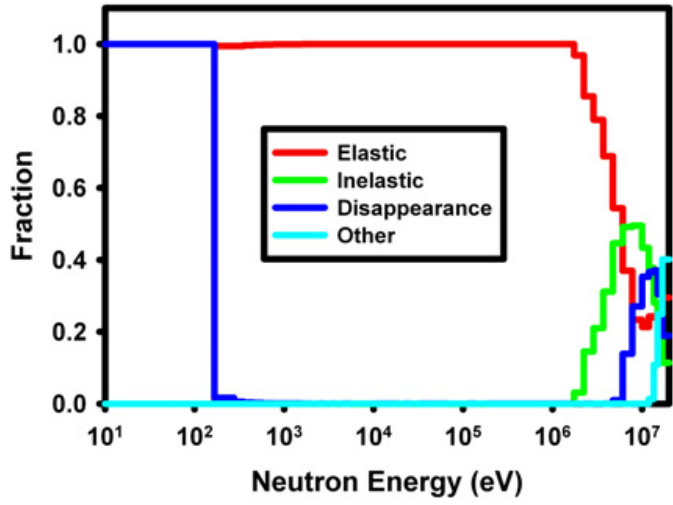

Figure 1. Fractional contribution of the various ${ }^{28} \mathrm{Si}$ damage components.

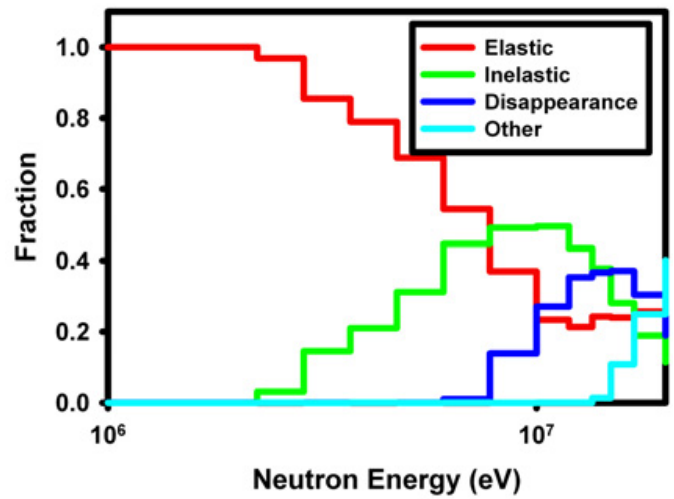

Figure 2. Fractional contribution in the high neutron energy region for the ${ }^{28} \mathrm{Si}$ damage components.

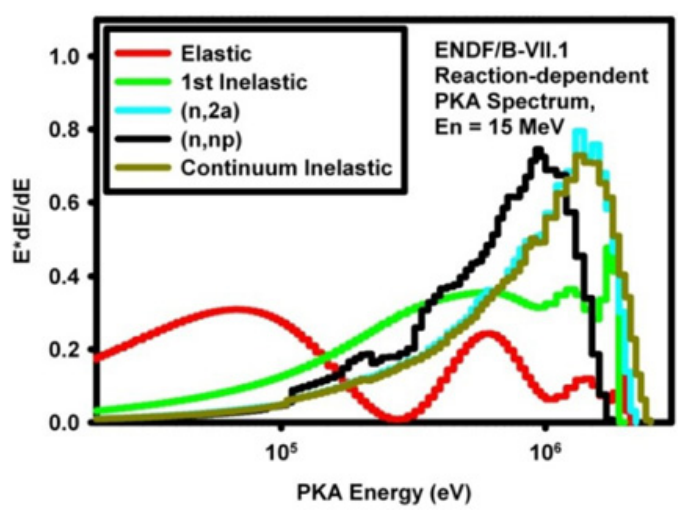

Figure 3. Reaction-dependent recoil spectra for $15-\mathrm{MeV}$ neutron.

in the elastic channel, but there can be significant differences for some of the disappearance channels for neutron energies near the reaction threshold energy. The uncertainty treatment needs to reflect the extend to which the uncertainty in the recoil spectra affects the displacement damage energy.

\subsection{Uncertainty propagation}

Because of the nonlinear dependence of the damage energy, seen in Eq. (1), on the cross section and recoil atom spectra terms, a rigorous treatment of the damage energy uncertainty requires that one use a Monte Carlo based approach. In order to perform a Monte Carlo sampling of the cross section, we looked to the TENDL-2015

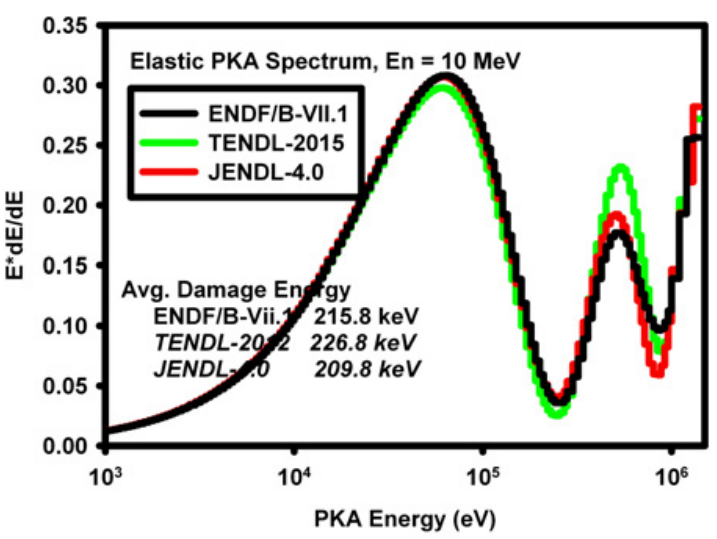

Figure 4. Variation seen in the elastic recoil spectra between different evaluations for a $10-\mathrm{MeV}$ neutron.

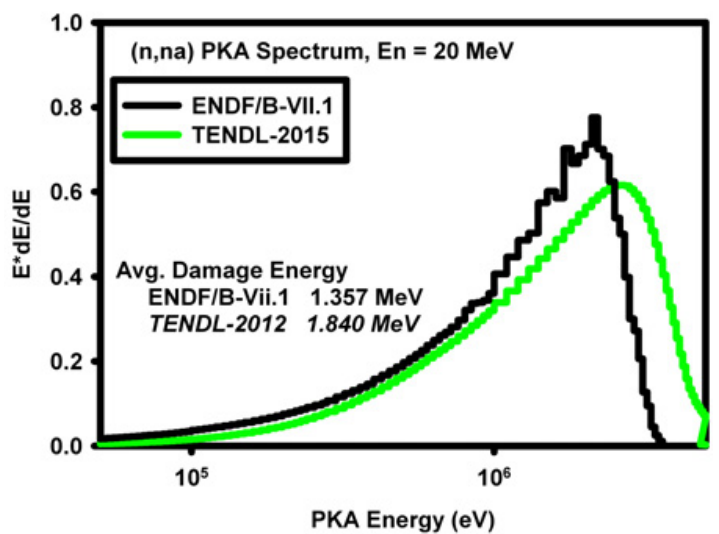

Figure 5. Variation seen in the $(\mathrm{n}, \mathrm{n} \alpha)$ recoil spectra between different evaluations for a $20-\mathrm{MeV}$ neutron.

cross section evaluation library [9] which is based on the TALYS system of nuclear reaction codes [10]. Since two of the authors (DR and AK) lead the TENDL-2015 team, we were able to obtain a 297 element random samplings for the nuclear data for the ${ }^{28} \mathrm{Si}$ isotope. This permitted us to use a Total Monte Carlo (TMC) [3] approach to treat this nonlinear propagation of uncertainty through the equation that defines the damage energy. We used an 89-group neutron energy bin structure in the NJOY-2012 code to generate a 297-element set of sharp threshold damage energies from the random TENDL-2015 evaluations, and then computed the energy-dependent covariance matrix that corresponded to this set of damage energies. Figure 6 shows the resulting standard deviation and Fig. 7 shows the correlation matrix for the total sharp threshold displacement damage energy. A combination of the standard deviation and correlation matrix is sufficient to define the covariance matrix that provides a high fidelity uncertainty characterization due of the uncertainty in the nuclear data. The analysis was carried out for each of the previously defined damage energy components. Figures 8, 9, 10, and 11 show the correlation matrices for the elastic, inelastic, disappearance, and "other" components of the damage energy.

Figures 12 and 13 show the energy-dependence of the standard deviation for the various damage energy components. The red curve represents the uncertainty for the total damage energy. It clearly shows in the figures at neutron energies above $\sim 5 \mathrm{MeV}$. At lower energies, 


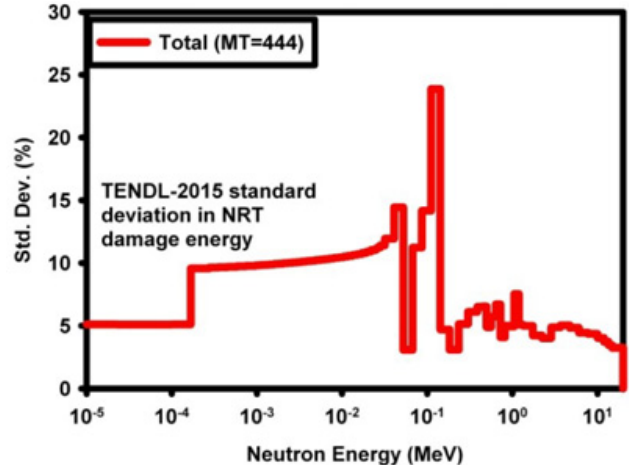

Figure 6. Energy-dependent standard deviation of the total sharp threshold damage energy for ${ }^{28} \mathrm{Si}$.

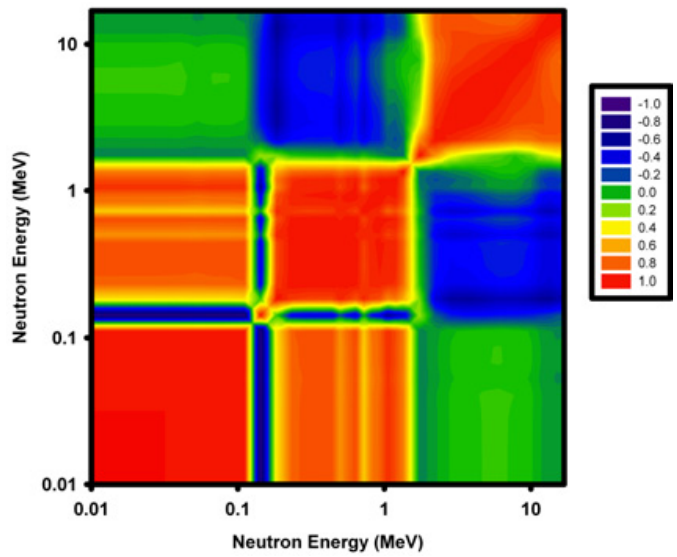

Figure 7. Energy-dependent correlation matrix for the total sharp threshold damage energy for ${ }^{28} \mathrm{Si}$.

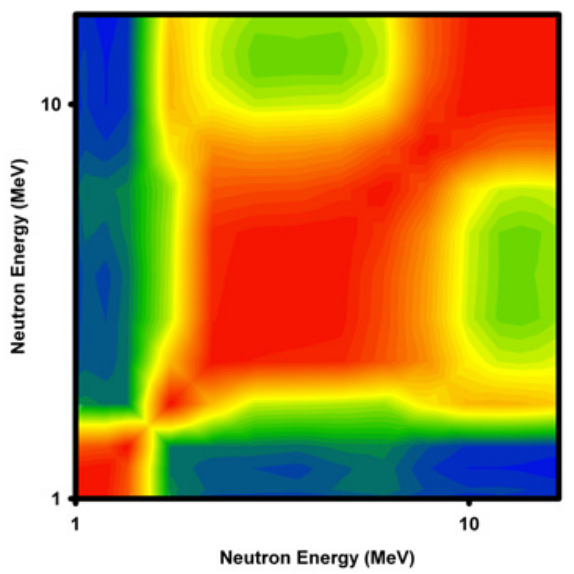

Figure 8. Energy-dependent correlation matrix for the elastic component of the sharp threshold damage energy for ${ }^{28} \mathrm{Si}$.

the curve for the total uncertainty merges first with that for the elastic (green) uncertainty component and then with that for the disappearance (black) uncertainty component. One immediately notes that, while the total damage energy is the sum of the various component damage energies, at high energy the uncertainty in the total damage energy is significantly smaller than the uncertainty for any of the individual components. This indicates that there is a strong correlation between the various damage energy components, a correlation that cannot be neglected

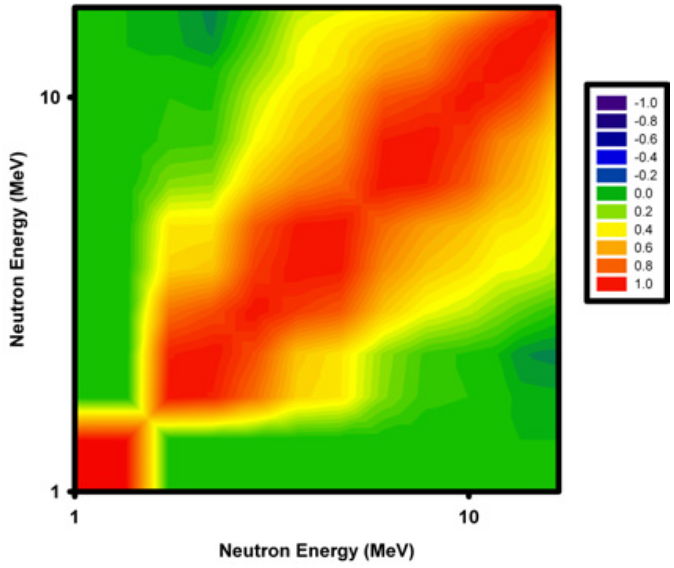

Figure 9. Energy-dependent correlation matrix for the inelastic component of the sharp threshold damage energy for ${ }^{28} \mathrm{Si}$.

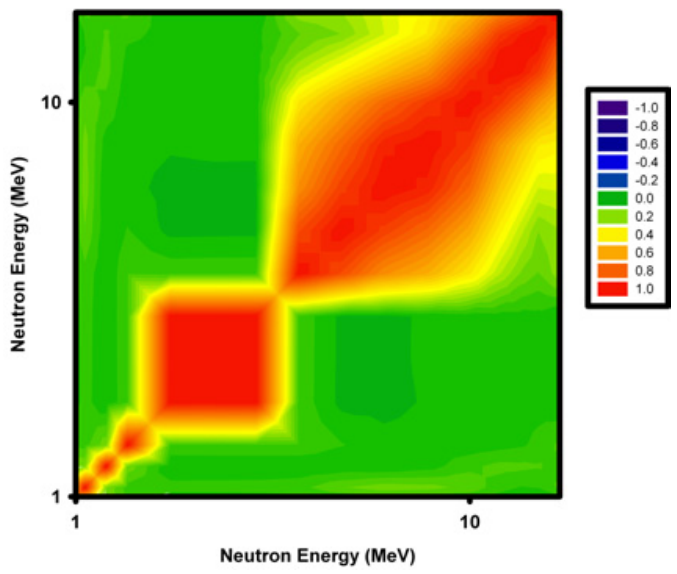

Figure 10. Energy-dependent correlation matrix for the disappearance component of the total sharp threshold damage energy for ${ }^{28} \mathrm{Si}$.

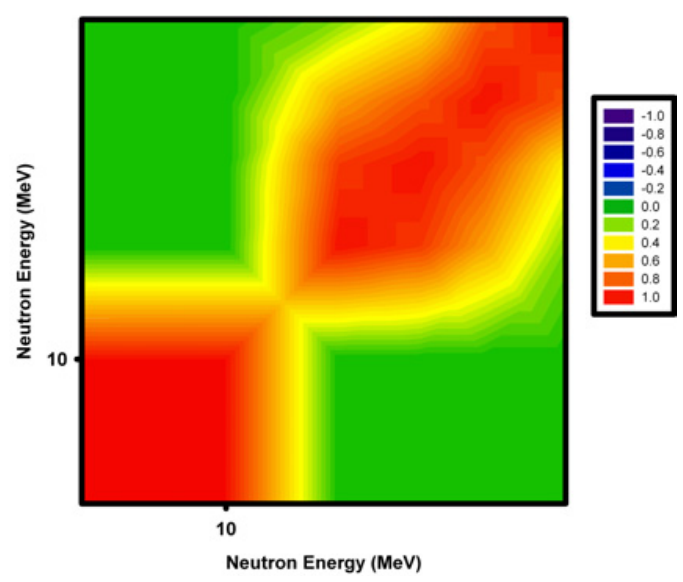

Figure 11. Energy-dependent correlation matrix for the "other" component of the total sharp threshold damage energy for ${ }^{28} \mathrm{Si}$.

in characterizing the uncertainty in the total damage energy.

This strong correlation at high neutron energies was further confirmed by examining a selection of the individual random draws from the TENDL-2015 cross section libraries. The variation in the total damage energy seen in this subset of the sample draws was very small while, consistent with Fig. 11, a much larger uncertainty 


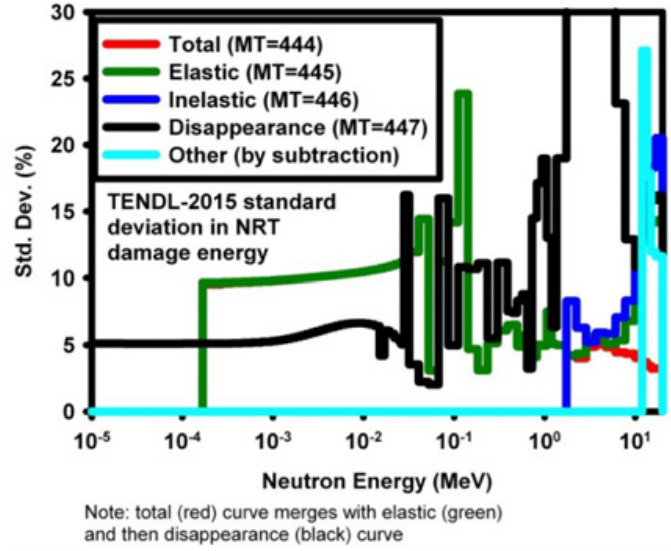

Figure 12. Energy-dependent standard deviation of the various components of the total sharp threshold damage energy for ${ }^{28} \mathrm{Si}$.

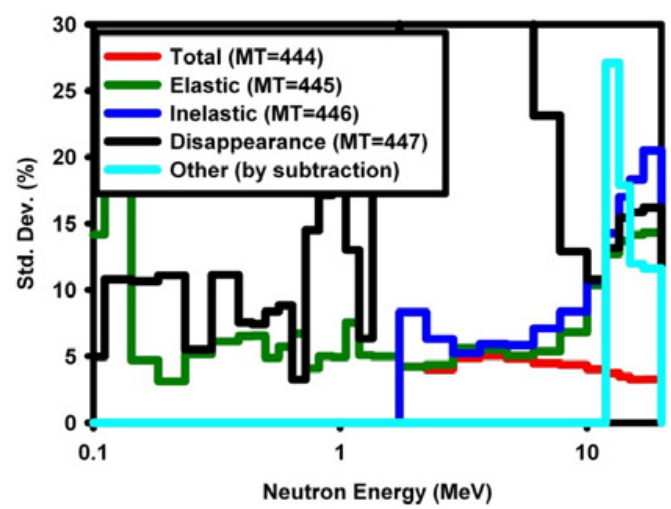

Figure 13. Expanded view of the energy-dependent standard deviation of the various components of the total sharp threshold damage energy for ${ }^{28} \mathrm{Si}$.

was seen in the other damage energy components. No subset of the individual damage energy components stood out as being strongly correlated, rather, it was the ensemble of damage energy contributions from the separate components that tended to cancel out the variation seen in the total damage energy.

This correlation seen between the uncertainty contributions for the different reaction channels clearly demonstrates that only a sample-based, i.e., Monte Carlo, nonlinear propagation of uncertainty that fully incorporates the underlying physics-based correlations between the different reaction channels, like that provided by the TMC approach, is adequate for the uncertainty analysis.

An examination of the TMC-produced energydependent damage energies showed that, while the distributions at low energies were normally distributed (Gaussian), at higher energies there was a significant skewness in the distributions. Since the covariance representation does not capture the higher moments of a distribution, the use of an energy-dependent damage energy, even in conjunction with a covariance propagation of the uncertainty, is less rigorous than the use of a TMC approach all the way through to the integral damage metric.
Table 1. Variation in damage energy due exclusively to the shape of the recoil spectrum.

\begin{tabular}{|c|c|c|}
\hline $\begin{array}{c}\text { Reaction } \\
\text { Channel }\end{array}$ & $\begin{array}{c}\text { Neutron } \\
\text { Energy } \\
(\mathrm{MeV})\end{array}$ & $\begin{array}{c}\text { Percent Non- } \\
\text { ionizing Energy } \\
\text { from PKA Spectrum }\end{array}$ \\
\hline & 0.0125 & $6.8007 \pm 0.007 \%$ \\
Elastic & 0.125 & $6.1318 \pm 0.477 \%$ \\
& 1.5 & $4.6274 \pm 2.791 \%$ \\
& 10.5 & $2.4360 \pm 14.34 \%$ \\
& 19.5 & $1.3679 \pm 17.791 \%$ \\
& 2.5 & $4.401 \pm 0.428 \%$ \\
$1^{\text {st }}$ Inelastic & 5.5 & $5.5502 \pm 0.204 \%$ \\
& 10.5 & $6.2711 \pm 1.202 \%$ \\
& 10.5 & $4.8633 \pm 5.937 \%$ \\
\hline & 3.5 & $6.9561 \pm 0.835 \%$ \\
$(\mathrm{n}, \alpha)$ & 5.5 & $9.9629 \pm 0.241 \%$ \\
& 10.5 & $10.5656 \pm 1.971 \%$ \\
& 14.5 & $9.1474 \pm 4.052 \%$ \\
& 19.5 & $8.6840 \pm 2.560 \%$ \\
\hline & 4.5 & $3.9973 \pm 0.188 \%$ \\
$(\mathrm{n}, \mathrm{p})$ & 5.5 & $4.3046 \pm 0.37 \%$ \\
& 10.5 & $4.7562 \pm 1.75 \%$ \\
& 14.5 & $5.0490 \pm 2.11 \%$ \\
& 19.5 & $4.7962 \pm 4.67 \%$ \\
\hline & 14.5 & $5.857 \pm 0.70 \%$ \\
$(\mathrm{n}, \mathrm{n} \alpha)$ & 17.5 & $6.644 \pm 1.9 \%$ \\
& 19.5 & $6.575 \pm 1.5 \%$ \\
\hline & 12.5 & $2.105 \pm 0.57 \%$ \\
& 14.5 & $3.880 \pm 2.2 \%$ \\
& 17.5 & $4.187 \pm 2.65 \%$ \\
& 19.5 & $4.315 \pm 2.97 \%$ \\
\hline
\end{tabular}

An analysis was made, using a subset of 297-element random TENDL-2015 nuclear evaluations, to separate the effect of the variation in the magnitude of the cross section from the shape of the recoil spectrum. The recoil spectra were extracted from the evaluation, normalized to unit fluence, and then the damage energy for the primary recoil atom was computed. Table 1 shows that the variation in the elastic channel damage energy due to the shape of the recoil spectrum is small for low incident neutron energies but grows with increasing neutron energy. This is consistent with the overall combined trend seen for the elastic channel in Fig. 13. The percent of energy going into the damage energy is seen to decrease with increasing incident neutron energy since the recoil atom energy increases and the Robinson damage partition function indicates that a smaller fraction of the recoil atom energy goes into displacements, as opposed to ionization, with increasing ion energy.

The variation in Table 1 for the $1^{\text {st }}$ inelastic reaction channel damage energy due to the shape of the recoil spectrum is smaller than the overall standard deviation shown in Fig. 13 for the total inelastic channel. This could mean that more of the observed variation came from the other discrete inelastic channels and/or the continuum inelastic channel (since Fig. 13 is for the total inelastic contribution). Alternately, this might indicate that more of the variation seen in Fig. 13 is due to the variation in the magnitude of the cross section as opposed to the shape of the recoil spectrum.

The variation in the Table $1(\mathrm{n}, \mathrm{n} \alpha)$ and $(\mathrm{n}, \mathrm{np})$ reaction channel damage energies is seen to be significantly smaller 
than that shown for the "other" damage energy component in Fig. 13. Since these two reaction channels are two of the dominant contributors to the "other" damage energy, this suggests that the major contributor to the standard deviation seen in Fig. 13 for this "other" damage energy component comes from the variation in the magnitude of the cross section rather than in the variation in the energy-dependent shape of the recoil spectrum. This same observation applies to the (n,p) and (n,a) disappearance channels when comparing the Table 1 recoil energy shape contribution to damage energy with the total variation in Fig. 13.

\section{Conclusion}

This paper has examined the effect of the uncertainty in the nuclear data on the silicon displacement damage metric. The variation in the nuclear data was captured by using a TMC approach based on the random cross section evaluations available as part of the TENDL-2015 library. A strong correlation was found between the contributions to the damage energy from the various reaction channels. The TMC approach to the uncertainty characterization enabled us to rigorously capture all of the underlying correlations due to the nuclear data. The uncertainty contribution from the nuclear data was captured in the form of an energy-dependent covariance matrix and this covariance matrix can be used in subsequent analysis to characterize the uncertainty from primary radiation damage in silicon semiconductor materials that affects the change in minority carrier lifetime.

This work was supported in part by the U.S. Department of Energy under contract DE-AC04-94AL85000. Sandia National Laboratories is a multi-mission laboratory managed and operated by Sandia Corporation, a wholly owned subsidiary of Lockheed Martin Corporation, for the U.S. Department of Energy's National Nuclear Security Administration under contract DEAC04-94AL85000. This work was funded in part by Sandias Laboratory Directed Research and Development Program (Project No. 173104).

\section{References}

[1] ASTM E722-14, Standard Practice for Characterizing Neutron Fluence Spectra in Terms of an Equivalent Monoenergetic Neutron Fluence for Radiation-Hardness Testing of Electronics, ASTM International, West Conshohocken, PA (2014)

[2] ASTM E1855-10, Standard Test Method for Use of 2N2222A Silicon Bipolar Transistors as Neutron Spectrum Sensors and Displacement Damage Monitors, ASTM International, West Conshohocken, PA (2014)

[3] D. Rochman, A. Koning, et al., "Nuclear Data Uncertainty Propagation: Total Monte Carlo vs. Covariances," Journal of Korean Physics 59, 12361241 (2011)

[4] P.J. Griffin, Relationship between Metrics used to Represent Displacement Damage in Materials, report SAND-2014-3341, Sandia National Laboratories, Albuquerque, NM (April, 2014)

[5] G.H. Kinchin, R.S. Pease, "The Displacement of Atoms in Solids by Radiation", Reports on Progress in Physics 18 (1955)

[6] M.J. Norgett. M.T. Robinson, I.M. Torrens, "A Proposed Method of Calculating Displacement Dose Rates," Nuclear Engineering and Design 33, 50-54 (1975)

[7] The NJOY Nuclear Data Processing System, Version, R.E. MacFarlane, D.M. Muir, R.M. Boicourt, A.C. Kahler, report LA-UR-12-27079, Los Alamos National Laboratory, Los Alamos, NM (Dec. 20, 2012)

[8] J. Lindhard, M. Scharff, H. Schiott, "Range Concepts and Heavy Ion Ranges," Mat. Phys. Medd. Dan. Vld. Selsk 33, 1-40 (1963)

[9] "TENDL-2015: TALYS-based evaluated nuclear data library", A.J. Koning, D. Rochman, J. Kopecky, et al., https://tendl.web.psi.ch/tendl_2015/ tend12015

[10] A.J. Koning, D. Rochman, Modern Nuclear Data Evaluation with the TALYS Code System, Nuclear Data Sheets 113, 2841-2934 (2012) 